\title{
Leukemia cells demonstrate a different metabolic perturbation provoked by 2-deoxyglucose
}

\author{
HIROSHI MIWA $^{1}$, MASATO SHIKAMI ${ }^{1}$, MINEAKI GOTO ${ }^{1}$, SHOHEI MIZUNO ${ }^{1}$, MIYUKI TAKAHASHI ${ }^{1}$, \\ NORIKAZU TSUNEKAWA-IMAI ${ }^{1}$, TAKAMASA ISHIKAWA ${ }^{2}$, MOTONORI MIZUTANI ${ }^{1}$, TOMOHIRO HORIO ${ }^{1}$, \\ MAYUKO GOTOU $^{1}$, HIDESUKE YAMAMOTO ${ }^{1}$, MOTOHIRO WAKABAYASHI $^{1}$, MASAYA WATARAI ${ }^{1}$, \\ ICHIRO HANAMURA ${ }^{1}$, AKIRA IMAMURA ${ }^{1}$, HIDETSUGU MIHARA $^{1}$ and MASAKAZU NITTA ${ }^{1}$ \\ ${ }^{1}$ Department of Internal Medicine, Division of Hematology, Aichi Medical University School of Medicine, \\ Nagakute, Aichi; ${ }^{2}$ Human Metabolome Technologies, Tsuruoka, Yamagata, Japan
}

Received December 4, 2012; Accepted January 30, 2013

DOI: $10.3892 /$ or.2013.2299

\begin{abstract}
The shift in energy metabolism from oxidative phosphorylation to glycolysis can serve as a target for the inhibition of cancer growth. Here, we examined the metabolic changes induced by 2-deoxyglucose (2-DG), a glycolysis inhibitor, in leukemia cells by metabolome analysis. NB4 cells mainly utilized glucose as an energy source by glycolysis and oxidative phosphorylation in mitochondria, since metabolites in the glycolytic pathway and in the tricarboxylic acid (TCA) cycle were significantly decreased by 2-DG. In THP-1 cells, metabolites in the TCA cycle were not decreased to the same extent by 2-DG as in NB4 cells, which indicates that THP-1 utilizes energy sources other than glucose. TCA cycle metabolites in THP-1 cells may be derived from acetyl-CoA by fatty acid $\beta$-oxidation, which was supported by abundant detection of carnitine and acetylcarnitine in THP-1 cells. 2-DG treatment increased the levels of pentose phosphate pathway (PPP) metabolites and augmented the generation of NADPH by glucose-6-phosphate dehydrogenase. An increase in NADPH and upregulation of glutathione synthetase expression resulted in the increase in the reduced form of glutathione by 2-DG in NB4 cells. We demonstrated that a combination of 2-DG and inhibition of PPP by dehydroepiandrosterone (DHEA) effectively suppressed the growth of NB4 cells. The replenishment of the TCA cycle by fatty acid oxidation by carnitine palmitoyltransferase in THP-1 cells, treated by 2-DG, might be regulated by AMPK, as the combination of 2-DG and inhibition of AMPK by compound $\mathrm{C}$ potently suppressed the growth of THP-1 cells. Although 2-DG has been effective in
\end{abstract}

Correspondence to: Dr Hiroshi Miwa, Department of Internal Medicine, Division of Hematology, Aichi Medical University School of Medicine, Nagakute, Aichi 480-1195, Japan

E-mail: hmiwa@aichi-med-u.ac.jp

Key words: acute myelogenous leukemia, 2-deoxyglucose, metabolome, glycolysis, pentose phosphate pathway, glutathione, carnitine preclinical and clinical studies, this treatment has not been fully explored due to concerns related to potential toxicities such as brain toxicity at high doses. We demonstrated that a combination of 2-DG and DHEA or compound $\mathrm{C}$ at a relatively low concentration effectively inhibits the growth of NB4 and THP-1 cells, respectively. These observations may aid in the identification of appropriate combinations of metabolic inhibitors at low concentrations which do not cause toxicities.

\section{Introduction}

One of the fundamental changes that occurs in cancer cells is the shift in energy metabolism from the generation of ATP from oxidative phosphorylation to glycolysis even in the presence of sufficient oxygen (Warburg effect) $(1,2)$. Several agents that specifically inhibit glycolytic metabolism, such as 2-deoxy-D-glucose (2-DG), have been used as effective anticancer agents in cellular systems and in animal models $(3,4)$. Similar to glucose, 2-DG is taken up through glucose transporters (GLUTs) and is phosphorylated by hexokinase (HK) to form 2-DG-6-phosphate (2-DG-6-P). 2-DG-6-P accumulates within the cell and is not metabolized further. Then, 2-DG-6-P induces cell growth arrest and cell death by inhibiting 2 glycolytic enzymes, HK and phosphoglucose isomerase (PGI) $(5,6)$.

Although 2-DG has been undergoing clinical trials for treatment of several types of cancers, its efficacy as a monotherapy is limited by systemic toxicity at high doses (7-9). However, 2-DG can sensitize tumors to other chemotherapeutic agents or radiotherapy $(10,11)$.

Here, we examined the metabolic changes induced by 2-DG in leukemia cells by metabolome analysis, and aimed to identify the critical metabolic pathway which can be targeted in conjunction with glycolysis inhibition.

\section{Materials and methods}

Cell lines. Two acute myelogenous leukemia (AML) cell lines were used in this study. NB4, a t(15;17) APL cell line, was provided by Dr M. Lanotte (Saint Louis Hospital, Paris, France). THP-1, a monocytic AML monocytic cell line 


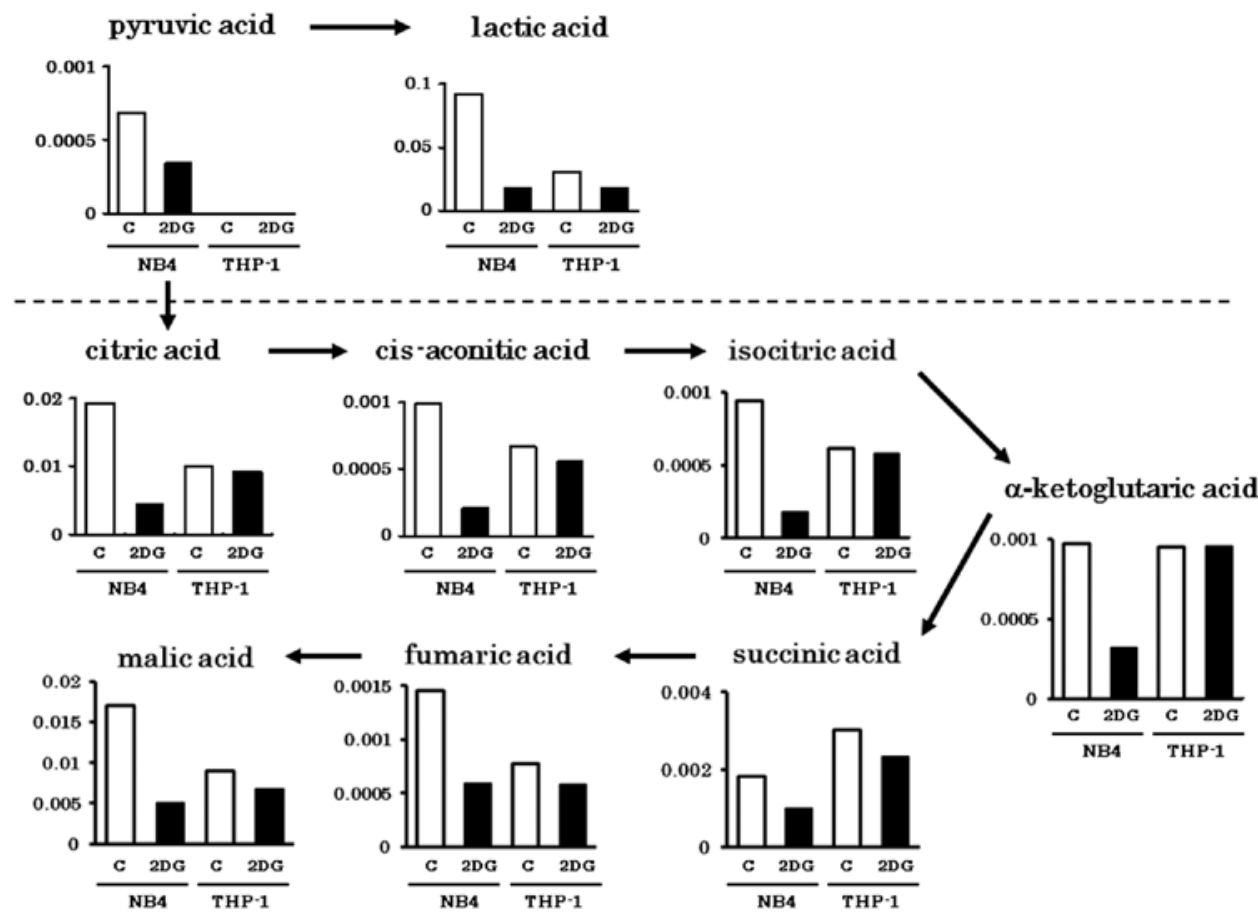

Figure 1. NB4 or THP-1 cells were cultured in RPMI-1640 containing 3\% FCS with or without the glycolysis inhibitor, 2-DG (0.5 mM), for 24 h. The cells were subsequently analyzed for intracellular metabolite levels of the glycolytic pathway and TCA cycle by capillary electrophoresis time-of-flight mass spectrometry (CE-TOFMS). Data are expressed as the concentration of the metabolite $\left(\mathrm{pmol} / \mathrm{mm}^{3}\right)$. Dotted line indicates the mitochondrial membrane; the lower section represents inside the mitochondria. $\square$, control; $\mathbf{m}, 2$-DG treatment.

was obained from the Cell Resource Center for Biomedical Research (Tohoku University, Japan). Cell lines were grown in RPMI-1640 medium containing 10\% fetal calf serum (FCS, Thermo Electron, Melbourne, Australia) in a humidified atmosphere of $5 \% \mathrm{CO}_{2}$ and $95 \%$ air at $37^{\circ} \mathrm{C}$.

Metabolome analysis. The cell lines were cultured in RPMI-1640 containing 3\% FCS with or without the glycolysis inhibitor, 2-DG $(0.5 \mathrm{mM})$, for $24 \mathrm{~h}$. Then, $5 \times 10^{6}$ cells were centrifuged and washed in 5\% mannitol. After centrifugation and removal of mannitol, cells were suspended in methanol. The samples were analyzed by capillary electrophoresis timeof-flight mass spectrometry (CE-TOFMS) (12).

Glutathione measurement. After a 24-h culture with or without 2-DG, cells were collected and assayed for glutathione (GSH) content using a GSSG/GSH quantification kit (Dojindo, Japan).

Cell cultures. Cell lines were cultured in RPMI-1640 containing $3 \%$ FCS with the glycolysis inhibitor, 2-DG $(0.5 \mathrm{mM})$, the inhibitor of G6P dehydrogenase [first step of the pentose phosphate pathway (PPP)], dehydroepiandrosterone (DHEA) $(20 \mu \mathrm{M})$, AMPK inhibitor compound $\mathrm{C}(1 \mu \mathrm{M})$, or in combination for 48 h. MTS [3-(4,5-dimethylthiazol-2-yl)-5-(3-carboxymethoxyphenyl)-2-(4-sulfophenyl)-2H-tetrazolium] (Promega, Madison, WI, USA) was then added to each culture. After 3 additional hours of incubation, absorbance at $490 \mathrm{~nm}$ was measured by an ELISA plate reader. Cell lines were also cultured in RPMI-1640 containing 3\% FCS with or without DHEA (20 $\mu \mathrm{M})$ and the GSH synthesis inhibitor, buthylsulfoximine (BSO) $(20 \mu \mathrm{M})$ for $48 \mathrm{~h}$. Then, MTS assay was also carried out.
Quantitative real-time PCR. Total RNA was isolated using the RNeasy Mini kit (Qiagen, Germany). Random hexamer priming and PrimeScript reverse transcriptase (Takara, Japan) were used to generate cDNA. Real-time reverse transcriptase polymerase chain reaction (RT-PCR) was carried out using a StepOne Plus Real-Time PCR system (Applied Biosystems, USA). Primers for PCR were as follows: glutathione synthetase forward, 5'-CCCTGCCCGAGTGGTCCAGT-3'; reverse, 5'-CACTCCCGCTGCCACACCAC-3' and 18S rRNA (as a control gene) forward, 5'-CGGCGACGACCCATTCGAAC-3'; reverse, 5'-GAATCGAACCCTGATTCCCCGTC-3'. The relative gene expression level was determined by comparison with 18S rRNA.

Statistical analysis. Statistical analysis was carried out by the t-test to examine the difference in growth of the cell lines, GSH content or glutathione synthetase mRNA expression.

\section{Results}

Metabolome analysis of the leukemia cell lines. Metabolites in the glycolytic pathway (pyruvic acid and lactic acid) were abundantly detected in the NB4 cells. However, these were greatly decreased by 2-DG treatment (Fig. 1). Metabolites of the tricarboxylic acid (TCA) cycle were comparably detected in both cell lines. The amounts of TCA cycle metabolites in the THP-1 cells were not obviously influenced by 2-DG as in the NB4 cells. This finding indicates that TCA cycle metabolites in the THP-1 cells were derived from non-glucose materials such as amino acids or fatty acids. Metabolites of PPP (Ru5P, R5P, PRPP) were abundantly detected in both NB4 and THP-1 cells, particularly when treated with 2-DG 
A

ribulose 5-phosphate

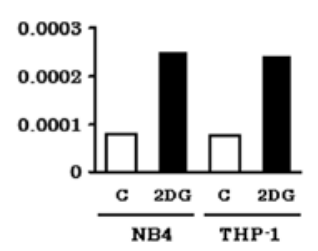

B ribose 5-phosphate

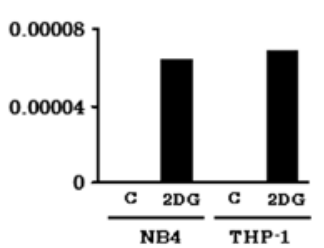

PRPP

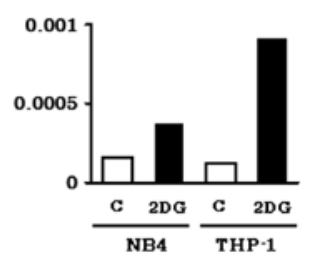

carnitine

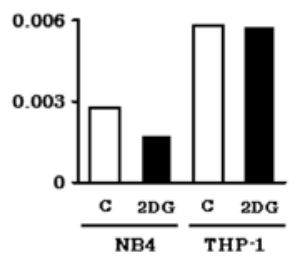

acetylcarnitine

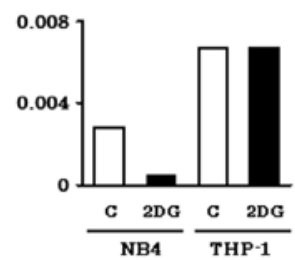

Figure 2. (A) Intracellular metabolite levels of the pentose phosphate pathway (PPP) in the NB4 and THP-1 cells as analyzed by CE-TOFMS. (B) Intracellular levels of carnitine and acetylcarnitine, used for fatty acid incorporation into mitochondria, in the NB4 and THP-1 cells as analyzed by CE-TOFMS. $\square$, control; a, 2-DG treatment.

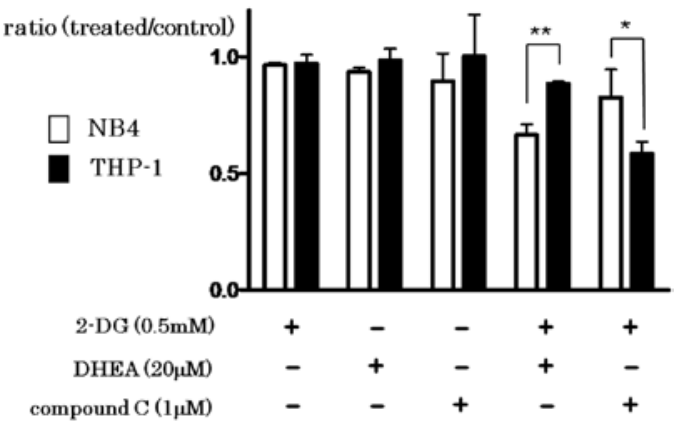

Figure 3. Growth inhibition of the leukemia cell lines (NB4, THP-1) by 2-DG (glycolysis inhibitor), DHEA (G6P dehydrogenase inhibitor), compound C (AMPK inhibitor) or in combination. MTS counts following a 48 -h culture with treatment divided by the MTS counts of the control (average of triplicate experiments) are shown. Simultaneous addition of 2-DG and DHEA inhibited the growth of NB4 cells $\left({ }^{* *} \mathrm{P}<0.01\right)$, while simultaneous addition of 2-DG and compound $\mathrm{C}$ inhibited the growth of THP-1 cells more effectively than that of the NB4 cells ( $\mathrm{P}<0.05)$.

(Fig. 2A). Carnitine and its acetylated form, acetylcarnitine are important for incorporation of fatty acids into mitochondria. Consistent with our previous observation that THP-1 depends on fatty acid oxidation for energy production (13), carnitine and acetylcarnitine were abundantly detected in THP-1 cells even with 2-DG treatment (Fig. 2B).

Synergistic effect of the inhibition of glycolysis and PPP or $A M P K$. The addition of 2-DG $(0.5 \mathrm{mM})$, DHEA $(20 \mu \mathrm{M})$ or compound $\mathrm{C}(1 \mu \mathrm{M})$ did not effectively inhibit the growth of both cell lines. However, simultaneous addition of 2-DG and DHEA synergistically inhibited the growth, particularly in the NB4 cells $(\mathrm{P}=0.0011)$, while simultaneous addition of 2-DG and compound $\mathrm{C}$ inhibited the growth of THP-1 cells more effectively than that of NB4 cells ( $\mathrm{P}=0.0319)$ (Fig. 3).

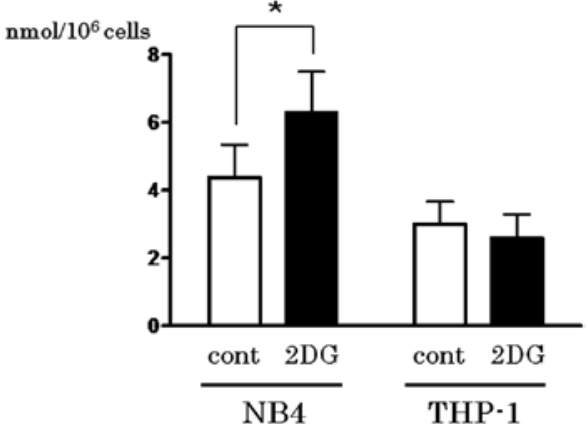

Figure 4. Content of the reduced form of GSH was measured after a 24-h culture with or without 2-DG $(0.5 \mathrm{mM})$. The reduced form of GSH was upregulated following 2-DG treatment only in the NB4 cells ( $(\mathrm{P}<0.05)$.

Activation of PPP by 2-DG treatment leads to reduction in $G S H$. The first step of PPP is triggered by G6P dehydrogenase, which produces NADPH. Since NADPH is utilized for GSH reduction, the content of the reduced form of GSH in the cell lines treated with or without 2-DG was determined. As shown in Fig. 4, the reduced form of GSH was upregulated following 2-DG treatment only in the NB4 cells $(\mathrm{P}=0.0494)$.

BSO inhibits the synthesis of GSH. DHEA inhibits G6P dehydrogenase, resulting in decreased NADPH and the reduced form of GSH. As shown in Fig. 5, the growth of NB4 cells was greatly inhibited by the addition of BSO $(\mathrm{P}=0.033)$, DHEA $(\mathrm{P}=0.0254)$ or in combination $(\mathrm{P}=0.0001)$ when compared with the effect in THP-1 cells.

Expression of glutathione synthetase is upregulated by 2-DG in NB4 cells. Glutathione synthetase (GS) catalyzes the condensation of $\gamma$-glutamylcysteine and glycine to form glutathione. As shown in Fig. 6, real-time quantitative RT-PCR study 


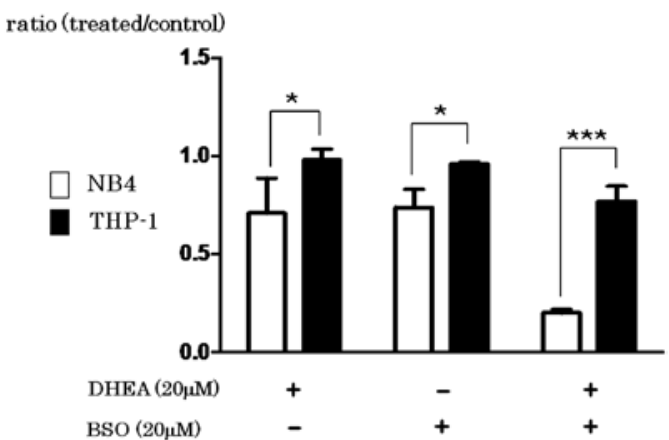

Figure 5. Growth inhibition of the leukemia cell lines (NB4, THP-1) by DHEA (G6P dehydrogenase inhibitor), BSO (GSH synthesis inhibitor) or in combination. MTS counts following a 48-h culture with treatment divided by the MTS counts of the control (average of triplicate experiments) are shown. For each treatment, the growth of NB4 cells was more potently inhibited than that of the THP-1 cells ( $\left.\mathrm{P}<0.05,{ }^{* * *} \mathrm{P}<0.001\right)$.

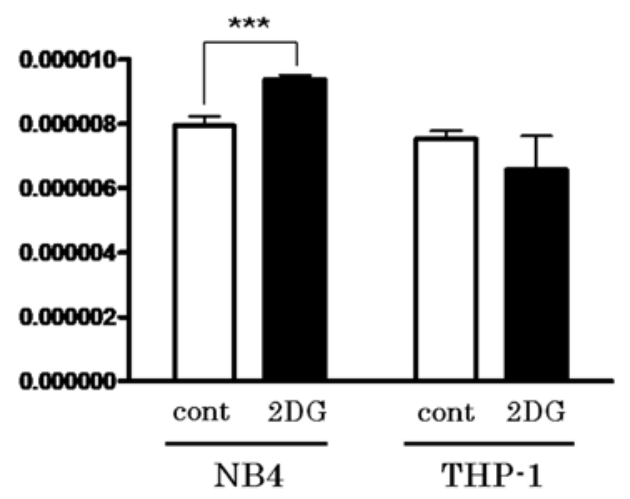

Figure 6. Real-time quantitative RT-PCR study reveales that expression of glutathione synthetase (GS) is upregulated by 2-DG treatment in NB4 cells $\left({ }^{* * *} \mathrm{P}<0.001\right)$.

revealed that expression of GS was upregulated following 2-DG treatment in NB4 cells $(\mathrm{P}=0.0007)$. This may explain the finding that the reduced form of GSH was upregulated by 2-DG in NB4 cells and that the growth of NB4 cells was more effectively inhibited by BSO.

\section{Discussion}

Metabolome analysis revealed that NB4 cells mainly utilized glucose as an energy source by glycolysis and oxidative phosphorylation in mitochondria, as metabolites in the glycolytic pathway and in the TCA cycle were significantly decreased by 2-DG, a glycolysis inhibitor. In THP-1 cells, metabolites in the TCA cycle were not decreased to the same extent by 2-DG as in the NB4 cells, which indicates that THP-1 cells utilized an energy source other than glucose. TCA cycle metabolites in THP-1 cells may be derived from acetyl-CoA by fatty acid $\beta$-oxidation, which was supported by abundant detection of carnitine and acetylcarnitine in the THP-1 cells (Fig. 2B). Our previous observation (13) that THP-1 depends on fatty acid oxidation for energy production also corroborates of this finding. 2-DG treatment increased the PPP metabolites in both cell lines. After entering the cell, 2-DG is phosphorylated

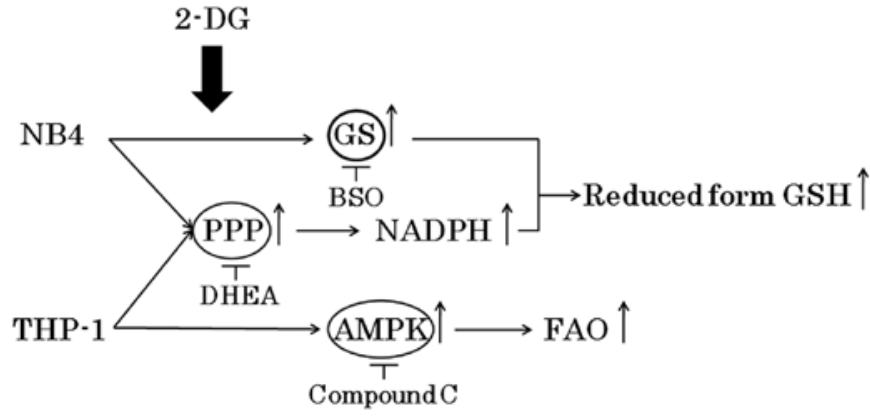

Figure 7. Following 2-deoxy-D-glucose (2-DG) treatment, the pentose phosphate pathway (PPP) is activated and NADPH production is upregulated by G6P dehydrogenase in both NB4 and THP-1 cells. Since glutathione synthetase (GS) is upregulated by 2-DG only in NB4 cells, the content of the reduced form of GSH is increased by 2-DG only in NB4 cells. We revealed that the combination of 2-DG and inhibition of PPP by DHEA or GS inhibition by buthylsulfoximine (BSO) effectively suppresses the growth of NB4 cells. In contrast, AMPK is activated by 2-DG treatment in THP-1 cells, resulting in activation of fatty acid oxidation to replenish the tricarboxylic acid (TCA) cycle. Use of both 2-DG and AMPK inhibitor, compound C, effectively inhibits the growth of THP-1 cells.

by hexokinase to form 2-DG-6-phosphate, which cannot be further metabolized, and its accumulation leads to inhibition of the glycolytic pathway and shunting through the PPP $(5,14,15)$. This PPP flux augments the generation of NADPH by glucose6-phosphate dehydrogenase (G-6-PDH). One of the uses of NADPH is to prevent oxidative stress by reducing glutathione (from GSSG to GSH). As shown in Fig. 4, an increase in the reduced form of GSH following 2-DG treatment was noted only in the NB4 cells. Upregulation of GS expression may explain the increase in the reduced form of GSH by 2-DG in the NB4 cells. We demonstrated that the combination of 2-DG and inhibition of PPP by DHEA effectively suppressed the growth of NB4 cells.

It has been reported that AMPK inhibits fatty acid synthesis and activates fatty acid oxidation $(16,17)$. We previously demonstrated that 2-DG treatment activates AMPK in THP-1 cells (13), which may explain the replenishment of the TCA cycle by fatty acid oxidation by carnitine palmitoyltransferase. Then, the combination of 2-DG and inhibition of AMPK by compound C potently suppressed the growth of THP-1 (Fig. 3).

Although 2-DG has been effective in preclinical and clinical studies, this treatment has not been fully explored due to concerns related to potential toxicities such as brain toxicity at high doses $(8,9)$. It is important to determine the appropriate combination of metabolic inhibitors at low concentrations which do not cause toxicities. Here, we demonstrated that the combination of 2-DG and DHEA or compound C at a relatively low concentration effectively inhibited the growth of NB4 and THP-1 cells, respectively (Fig. 7). Further studies are warranted to ascertain the efficacy and safety of these combinations.

\section{Acknowledgements}

We are grateful to Dr N. Kamada (Hiroshima University, Japan) for Kasumi-1 and to Dr M. Lanotte (Saint Louis Hospital, Paris, 
France) for the NB-4 cell line. We also thank Ms. A. Usui and A. Nakamura for their technical and secretarial assistance. This study was supported in part by the Ministry of Education, Culture, Sports, Science and Technology, Japan (MEXT)Supported Program for the Strategic Research Foundation at Private Universities, 2011-2015 (S1101027).

\section{References}

1. Warburg O: On the origin of cancer cells. Science 123: 309-314, 1956.

2. Gatenby RA and Gillies RJ: Why do cancers have high aerobic glycolysis? Nat Rev Cancer 4: 891-899, 2004.

3. Pelicano H, Martin DS, Xu RH and Huang P: Glycolysis inhibition for anticancer treatment. Oncogene 25: 4633-4646, 2006

4. El Mjiyad N, Caro-Maldonado A, Ramírez-Peinado S and Muñoz-Pinedo C: Sugar-free approaches to cancer cell killing. Oncogene 30: 253-264, 2011.

5. Sols A and Crane RK: Substrate specificity of brain hexokinase. J Biol Chem 210: 581-595, 1954.

6. Kurtoglu M, Gao N, Shang J, Maher JC, Lehrman MA, Wangpaichitr M, Savaraj N, Lane AN and Lampidis TJ: Under normoxia, 2-deoxy-D-glucose elicits cell death in select tumor types not by inhibition of glycolysis but by interfering with N-linked glycosylation. Mol Cancer Ther 6: 3049-3058, 2007.

7. Tennant DA, Durán RV and Gottlieb E: Targeting metabolic transformation for cancer therapy. Nat Rev Cancer 10: 267-277, 2010.

8. Porporato PE, Dhup S, Dadhich RK, Copetti T and Sonveaux P: Anticancer targets in the glycolytic metabolism of tumors: a comprehensive review. Front Pharmacol 2: 49, 2011.

9. Cheong H, Lu C, Lindsten T and Thompson CB: Therapeutic targets in cancer cell metabolism and autophagy. Nat Biotechnol 30: 671-678, 2012.
10. Maschek G, Savaraj N, Priebe W, Braunschweiger P, Hamilton K, Tidmarsh GF, De Young LR and Lampidis TJ: 2-Deoxy-Dglucose increases the efficacy of adriamycin and paclitaxel in human osteosarcoma and non-small cell lung cancers in vivo. Cancer Res 64: 31-34, 2004.

11. Singh D, Banerji AK, Dwarakanath BS, Tripathi RP, Gupta JP, Mathew TL, Ravindranath T and Jain V: Optimizing cancer radiotherapy with 2-deoxy-d-glucose dose escalation studies in patients with glioblastoma multiforme. Strahlenther Onkol 181: 507-514, 2005.

12. Ooga T, Sato H, Nagashima A, Sasaki K, Tomita M, Soga T and Ohashi Y: Metabolomic anatomy of an animal model revealing homeostatic imbalances in dyslipidaemia. Mol Biosyst 7: 1217-1223, 2011

13. Suganuma K, Miwa H, Imai N, Shikami M, Gotou M, Goto M, Mizuno S, Takahashi M, Yamamoto H, Hiramatsu A, Wakabayashi M, Watarai M, Hanamura I, Imamura A, Mihara H and Nitta M: Energy metabolism of leukemia cells: glycolysis versus oxidative phosphorylation. Leuk Lymphoma 51: 2112-2119, 2010

14. Chen W and Guéron M: The inhibition of bovine heart hexokinase by 2-deoxy-D-glucose-6-phosphate: characterization by ${ }^{31} \mathrm{P}$ NMR and metabolic implications. Biochimie 74: 867-873, 1992.

15. Sandulache VC, Ow TJ, Pickering CR, Frederick MJ, Zhou G, Fokt I, Davis-Malesevich M, Priebe W and Myers JN: Glucose, not glutamine, is the dominant energy source required for proliferation and survival of head and neck squamous carcinoma cells. Cancer 117: 2926-2938, 2011.

16. Hardie DG and Pan DA: Regulation of fatty acid synthesis and oxidation by the AMP-activated protein kinase. Biochem Soc Trans 30: 1064-1070, 2002.

17. Jeon SM, Chandel NS and Hay N: AMPK regulates NADPH homeostasis to promote tumour cell survival during energy stress. Nature 485: 661-665, 2012. 